\title{
SPRAY BEHAVIOR OF NON-NEWTONIAN FLUIDS: CORRELATION BETWEEN RHEOLOGICAL MEASUREMENTS AND DROPLETS/THREADS FORMATION
}

\author{
M. Negri, H. K. Ciezki, and S. Schlechtriem \\ DLR - German Aerospace Center \\ Institute of Space Propulsion \\ Lampoldshausen, Hardthausen 74239, Germany
}

\begin{abstract}
The formation of thread-like structures instead of droplets has been observed in recent studies on the injection of various gels and nonNewtonian fluids through a doublet impinging jet injector. In the present work, it has been studied if a correlation can be found between distinct fluid properties and thread formation. For this task, the tests have been conducted with different classes of fluids with various fluid properties. These classes are: Boger fluids, linear and crosslinked polymer solutions, and silica suspensions. The results show that a correlation exists between thread formation and the extensional behavior of the fluid, in particular, high values of the Trouton ratio. The characterization of the extensional behavior of fluids with a distinct yield stress has been conducted with a capillary rheometer, and the results obtained with this technique are compared to the spray behavior.
\end{abstract}

\section{INTRODUCTION}

Gelled fuels and propellants are of interest for rocket and ramjet propulsion systems because of their safety and performance benefits. One condition to achieve an efficient combustion in a limited combustor length is that the spray produced by the injector has a high specific surface area. Impinging jet injectors generally satisfy this requirement when used with low viscosity Newtonian fluids and sufficiently high injection velocities. The impact of the jets leads to the formation of fine droplets and, thus, the necessary high surface area of the droplet phase. Also, for gelled propellants, an impinging jet injector has been used successfully. An example is presented in Fig. $1 a$ where the breakup of an ethanol

This is an Open Access article distributed under the terms of the Creative Commons Attribution License 2.0, which permits unrestricted use, distribution, and reproduction in any medium, provided the original work is properly cited. 


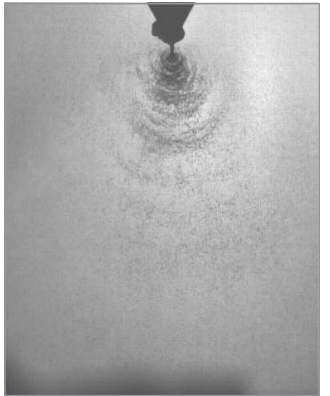

(a)

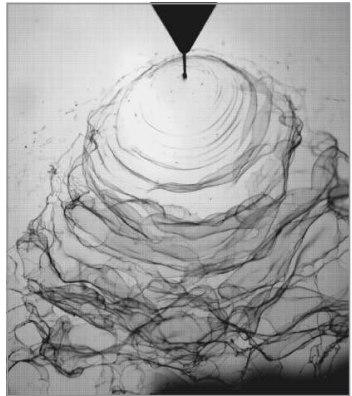

(b)

Figure 1 Comparison of droplets and threads formation: $(a)$ ethanol $+10 \% \mathrm{COK}$ $84, u_{j}=45.5 \mathrm{~m} / \mathrm{s}$; and $(b)$ water $+3.1 \%$ Methocel J12MS, $u_{j}=46.8 \mathrm{~m} / \mathrm{s}$

gel is shown. On the other hand, during the spray tests conducted at DLR in Lampoldshausen, it was observed that the atomization did not lead to the formation of droplets for several gels. Instead, thread-like structures $[1,2]$ are formed as shown in Fig. 1b. The specific surface area of these structures is much smaller compared to that of a droplet phase. The smaller specific surface area may lead to lower combustion efficiencies in a limited length combustor and the accumulation of unburned material on the wall of the combustion chamber.

The formation of thread-like structures with an impinging jet injector has also been observed in several other studies outside DLR. Miller et al. [3] studied a series of viscoelastic worm-like micelle solutions at injection velocities up to $10 \mathrm{~m} / \mathrm{s}$. They used a 60 degree impinging jet injector with an impinging distance of $1.5 \mathrm{~cm}$. The nozzles had a diameter of $1.5 \mathrm{~mm}$. These solutions did not lead to the formation of droplets when injected. Instead, for increasing injection velocities, two different structures were observed, which were called fluid webs and fluid tendrils. The analysis of the results was based on two nondimensional numbers: the elasticity number and the Weber number. The zero-shear-rate viscosity and the fluid relaxation time necessary to determine the elasticity number were determined through oscillatory tests with a rotational rheometer. The conclusion was that the elasticity delays the breakup of liquid filaments. In $[4,5]$, an impinging jet injector with orifice diameters of $0.406 \mathrm{~mm}$, an impingement angle of $120^{\circ}$, and injection velocities up to $35 \mathrm{~m} / \mathrm{s}$ have been used. The gels tested where linear and branched polymer solutions in mineral oil. A clear formation of threads was obtained with the linear polymer solutions. The spray formation behavior has been classified into regimes defined by ranges of elasticity number and injection velocity. The relaxation time $(\lambda)$ used to determine the value of the elasticity number was measured through oscillatory tests with a rotational rheometer. 
Besides the studies conducted with impinging jet injectors, the effect of nonNewtonian fluids on the spray behavior has also been studied with other types of injectors. The influence of non-Newtonian fluids on the suppression of droplets has been observed in several studies on laminar jets. Mun et al. [6] analyzed solutions of poly(ethylene oxide) (PEO) in a mixed solvent of glycerol and water. Polymers with different molecular weights were tested, from $8 \cdot 10^{3}$ to $1 \cdot 10^{6}$. These solutions had a shear viscosity that remained constant over a broad range of shear rates. Moreover, the composition was chosen so that all solutions had the same shear viscosity. The extensional properties of the fluids were characterized with a Rheometrics RFX opposed jet rheometer. The polymeric solutions with higher molecular weights showed a marked extensional thickening behavior: an increase in the apparent shear rate corresponded to a large increase in the Trouton ratio. It was found that the jet breakup is dominated by nonlinear effects due to high extensional stresses, related to the viscoelastic behavior of polymeric solutions. Polymeric solutions with high molecular weights did not break into drops as Newtonian fluids did. Instead, long threads of fluid connecting drops are formed. These are called in literature bead-on-string structures. Moreover the formation of satellite drops, typical of Newtonian fluids, was not observed for polymeric solutions. The studies from Christanti and Walker [7, 8] were a further development of the work conducted by Mun et al. [6]. They were focused mainly on the suppression of satellite drops. In these studies, the concept of fluid characteristic relaxation time was used to characterize the fluids. In [7], characteristic relaxation time was taken as the inverse of the critical extensional rates obtained by Mun et al. with an opposed jet rheometer. In [8], the relaxation times of the polymer solutions were measured through a free jet extensional rheometer. The fluid relaxation time increased only slightly by increasing the concentration of the polymer. On the other hand, an increase in the molecular weight of the polymer had a very strong effect in increasing the fluid relaxation time.

Antimisting (AM) agents are the polymers added to a fuel to prevent the formation of droplets. The most comprehensive paper on antimisting agents and their effect on spray behavior has been published by Chao et al. in 1984 [9]. Different polymeric solutes were tested, in particular, six different polyisobutylenes (PIB). An airblast atomizer was used. Considering a single kind of polymer, an increase of concentration increased the AM effectiveness. On the other hand, at a fixed concentration, the AM effectiveness increased with increasing molecular weights. For the most effective AM agents, the formation of droplets was completely suppressed, while the spray turned into a very large continuous filament.

Mansour and Chigier [10] studied the atomization of aqueous solutions of Xanthan gum and Polyacrilamide E10 with an airblast atomizer. For concentrations of the polymers above a certain level, the liquid did not atomize. Instead, long threads of liquid interspaced by droplets were formed. Extensional viscos- 
ity was measured with a capillary rheometer, using Binding theory to determine extensional properties from pressure drop measures. As indication of the applicability of the contraction flow analysis, a comparison was made between the vortex attachment length predicted by Binding's theory and the value determined from laser sheet flow visualization. It was found that this technique gives goods results only with relatively concentrated solutions. Solutions that lead to the formation of threads were characterized by a marked extensional thickening behavior.

Several studies have been conducted with polymer solutions injected through agricultural nozzles. Significant are, in particular, the works by Mun et al. [11], Thompson and Rothstein [12], Harrison et al. [13], and Stelter et al. [14]. These studies show the influence of the extensional behavior on hindering the formation of drops. To obtain a better understanding of the phenomenon of thread formation, a research campaign is currently ongoing at DLR. The first results were presented in [15].

The studies in literature show the influence of the elongational behavior on thread formation and droplet suppression. The measure of the extensional viscosity for a fluid with a distinct yield stress and relatively high viscosity is problematic. The gels used for propulsion application fall in this category. An opposed jet rheometer as the one used for the characterization of the Boger fluids in [6] can be used only if the viscosity of the fluid tested is relatively low. Also obtaining reliable results with capillary breakup extensional rheometry (CaBER) becomes more difficult when the fluid exhibits a distinct yield stress [16]. An apparatus that can be used with a very large range of fluids is the capillary rheometer. With this method, the extensional viscosity is determined by analyzing an entrance flow. The fluid is pushed from a reservoir tube through an orifice or a capillary and the entry pressure drop is recorded as function of the flow rate. When the fluid flows from the large reservoir tube into the orifice, the streamlines converge generating an extensional flow. It has to be mentioned that this flow is not a pure extensional flow because of the presence of the walls. The nonslip condition at the wall imposes a certain velocity profile on the fluid that adds a shear component to the flow. Different theories have been developed to separate shear and extensional effects. In the present work, the Cogswell theory has been used. This technique is the most widely used in literature (see the introduction to [17]). The superimposition of shear and extensional flow makes it difficult to obtain quantitative values of extensional viscosity with a capillary rheometer. On the other hand, as highlighted in [18], a capillary rheomether should allow the investigation of the extensional behavior of different fluids to be ranked.

Keeping in mind the limitation of such an apparatus, one of the objectives of the present work is to determine if a correlation exists between the values of elongational viscosity obtained with the capillary rheometer and the formation of threads. 


\section{THEORETICAL BACKGROUND: EXTENSIONAL FLOW AND EXTENSIONAL VISCOSITY OF A COMPLEX FLUID}

The fluids studied in the present work can be categorized as complex fluids. A complex fluid is a fluid in which two or more phases coexist. During the injection process, the flow has a strong extensional component. An extensional flow is a flow in which the elements of a fluid are stretched out or squeezed down rather than sheared [19]. The extensional viscosity is the resistance that a fluid offers to an extensional flow. For a Newtonian fluid, it can be shown theoretically that the value of the extensional viscosity is 3 times the shear viscosity. For distinct non-Newtonian fluids, the extensional viscosity can be significantly larger than the shear viscosity. This is due to the fact that in these fluids, normal forces can be generated. These forces increase the resistance of the fluid to an extensional flow. Thus, they increase the extensional viscosity. Often, the extensional behavior of a fluid is expressed in terms of the Trouton ratio $\left(\operatorname{Tr}=\eta_{E} / \eta\right)$ which is the ratio of extensional viscosity $\left(\eta_{E}\right)$ to shear viscosity $(\eta)$.

The behavior of a complex fluid in an extensional flow is influenced by the nature of the macromolecules or particles that are suspended in the fluid. A wellstudied case is a linear polymer solution in an extensional flow (see, for example, [20]). In an extensional flow, the molecules are stretched in the direction of the tensile stress as sketched in Fig. 2. The streamlines in the position indicated with $A$ in Fig. $2 b$ are more distant than in position $B$ and so, the fluid velocity will be higher in $B$ than in $A$. Each molecule will have an average velocity that is in-between the velocity of the fluid at its extremities. The more the chain elongates, the larger the difference in the fluid velocities at the two ends of the molecule. Thus, the molecule will create a drag on the surrounding fluid at both extremities: at one end, the molecule will be faster than the surrounding fluid and at the other end, it will be slower. Therefore, the presence of elongated molecules will create a normal force in the fluid and this leads to high Trouton ratios for linear polymer solutions. On the other hand, suspensions of particles with a limited aspect ratio will have smaller Trouton ratios.

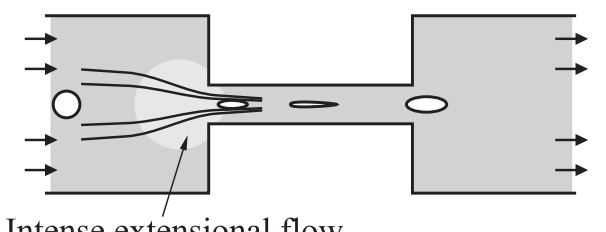

Intense extensional flow

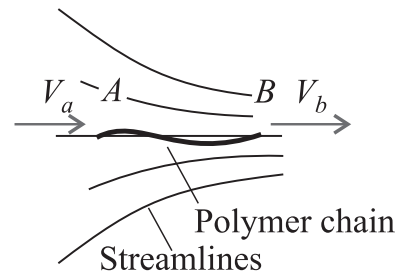

Streamlines

Figure 2 Extensional flow in a contraction (from [19]) (a) and linear polymer chain in an extensional flow $(b)$ 


\section{MATERIAL AND METHODS}

\subsection{Experimental Setup}

The experimental setup for the spray investigations consists of a cartridge with the fluid to be investigated, a hydraulic driving unit, and a modular injector unit (Fig. 3). The injector arms of the injector unit are mounted on movable rotary tables so that the impingement angle as well as the preimpingement length can be varied easily. The injector tips (nozzles) can easily be changed for the variation of the nozzle exit diameters and the internal injector geometry. For the present work, an impingement angle of $90^{\circ}$ and a preimpingement length of $10 \mathrm{~mm}$ were chosen. The diameter of the nozzles was $0.7 \mathrm{~mm}$. The high length-to-diameter ratio of the internal injector channel as well as the internal wall inclination angle of $20^{\circ}$ were chosen both to reduce the influences of separation by the formation of a vena contracta in the intake to the injector channel and to induce a more fully developed velocity profile at the injector exit. For the visualization of the spray patterns, the shadowgraph technique was used, together with two CCD (charge couple device) cameras, one parallel and one perpendicular to the plane of the injectors, and two Nanolite spark lights as light sources. Generally, more than 50 pictures were analyzed to ensure that stationary conditions were obtained for each experiment.

The rheological characterization of the fluids was conducted with a rotational and a capillary rheometers. The rotational rheometer was a Thermo Haake RS6000. The capillary rheometer was a Rosand 2000. The surface tension has been measured with a Krüss EasyDyne.

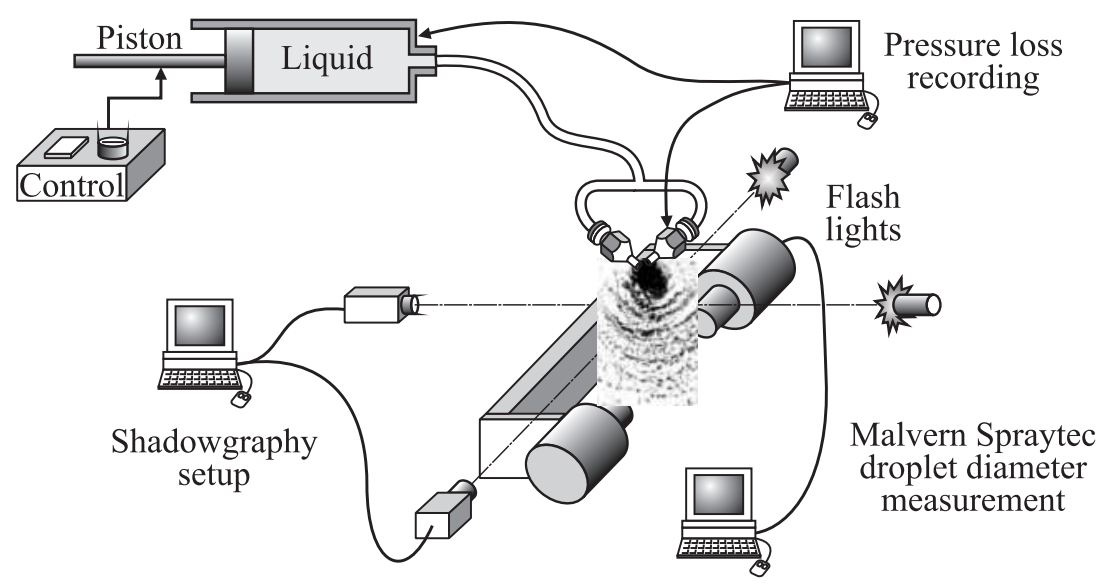

Figure 3 Spray tests experimental setup 


\subsection{Test Fluids}

The tests have been conducted with different classes of fluids. In Table 1, a summary of the materials used is given. Boger fluids are very diluted linear polymer solutions. They offer a significant advantage in comparison to more concentrated polymer solutions because they allow to separate elastic effects from viscous effects. More concentrated solutions are typically both shear thinning and extensional thickening. Thus, it is difficult to distinguish the influence of these two fluid properties on the flow. Boger fluids have a shear viscosity that does not vary significantly with the shear rate. Their extensional behavior is influenced by the molecular weight of the polymer. An increase of the molecular weight leads to a more marked extensional thickening behavior. The three Boger fluids used in the present work have the same composition as those used by Mun et al. [6]. They are the solutions of polyethylene oxide (PEO, Sigma Aldrich) in water and glycerol.

It could be very interesting to use linear polymers as gelling agents: very small amounts give a solution with a high yield stress. Most of the advantages of a gelled propellant are achieved only if the yield stress is high enough. Linear

Table 1 Composition of the test fluids

\begin{tabular}{|c|c|c|c|}
\hline \multicolumn{2}{|l|}{ Fluid } & \multicolumn{2}{|r|}{ Composition } \\
\hline & & Solvent & Solute \\
\hline \multirow{3}{*}{$\begin{array}{l}\text { Boger } \\
\text { fluids }\end{array}$} & BF1 & $\begin{array}{l}22.9 \% \text { glycerol } \\
69.875 \% \text { water }\end{array}$ & $1.1 \% \operatorname{PEO}\left(M_{w}=1 \cdot 10^{5} \mathrm{~g} / \mathrm{mol}\right)$ \\
\hline & BF2 & $\begin{array}{l}31.23 \% \text { glycerol } \\
68.5 \% \text { water }\end{array}$ & $0.27 \% \operatorname{PEO}\left(M_{w}=3 \cdot 10^{5} \mathrm{~g} / \mathrm{mol}\right)$ \\
\hline & BF3 & $\begin{array}{l}30 \% \text { glycerol } \\
69.875 \% \text { water }\end{array}$ & $0.125 \% \operatorname{PEO}\left(M_{w}=1 \cdot 10^{6} \mathrm{~g} / \mathrm{mol}\right)$ \\
\hline \multirow{6}{*}{$\begin{array}{l}\text { Linear } \\
\text { polymer } \\
\text { solutions }\end{array}$} & LP1 & Water & $0.5 \%$ Methocel J75MS $\left(M_{n}=220,000\right)$ \\
\hline & LP2 & Water & $1 \%$ Methocel J75MS $\left(M_{n}=220,000\right)$ \\
\hline & LP3 & Water & $2 \%$ Methocel J75MS $\left(M_{n}=220,000\right)$ \\
\hline & LP4 & Water & $2 \%$ Methocel F50 $\left(M_{n}=20,000\right)$ \\
\hline & LP5 & Water & $2 \%$ Methocel F4M $\left(M_{n}=86,000\right)$ \\
\hline & EG1 & Ethanol & $3.5 \%$ Methocel 311 \\
\hline $\begin{array}{l}\text { Crosslinked } \\
\text { polymer } \\
\text { solutions }\end{array}$ & CP1 & Water & 1\% Carbopol 980 \\
\hline Silica & PG1 & Paraffin & $4 \%$ Aerosil 200 \\
\hline suspensions & EG2 & Ethanol & $10 \%$ COK 84 \\
\hline
\end{tabular}

Remarks: $M_{w}$ is the average molecular weight and $M_{n}$ is the number average molecular weight. 
polymeric solutions are of particular interest in the present work because in previous research activities (see, for example, $[2,4]$ ) was observed that such solutions led to the formation of threads. The polymer solutions tested are the aqueous solutions of different kinds of Methocel (Dow Chemical Company). Methocel is the commercial name of methylcellulose, a linear polymer derivative from cellulose. It is available in different molecular weights; so, it is possible to test the influence of the same type of molecule with different molecular lengths. Experimental values of the extensional viscosity for the linear polymer solutions tested are not available in literature. The problems related with a direct measurement of the extensional viscosity of fluids with a distinct yield stress have been addressed in the introduction. However, applying the observations described in section 2 to linear polymer solutions, an increase in molecular weight is expected to lead to an increase in the Trouton ratio.

Beside linear polymers, also crosslinked polymers could be used as gelling agent. In this kind of solutions, the polymer molecules create chemical bonds between each other. This leads to a distinct yield stress even at low concentrations of the polymer. The crosslinked polymer solution tested here is an aqueous solution of Carbopol 980 (Lubrizol).

Silica suspensions are also often used as gelling agent. The structure of silica suspensions is made from branched aggregates of spherical particles as described in [21]. The size of the primary spherical particles for Aerosil, a kind of silica produced by Evonik, is $14 \mathrm{~nm}$. During the production process, the primary particles are fused together in aggregates that cannot be broken down by shear. The aggregates have a fractal, chain-like structure and generally, a modest aspect ratio [22]. When silica is dispersed in a suitable liquid, the aggregates can interact forming hydrogen bonds. This creates larger structures called flocs. The interaction of flocs creates a three-dimensional (3D) structure, leading to a gellike behavior of the suspension. These structures can be destroyed if the stress applied to the fluid is large enough. This causes the shear thinning behavior of flocculated silica suspensions.

As for linear polymer solutions, it is possible to consider the microscopic structure of the fluid to get an idea of the elongational behavior. The internal structure of silica suspensions is made of flocs, held together by hydrogen bonds. Once these structures are broken down during the injection process, the aggregates are left. The aggregates have a limited aspect ratio, and so, they will have a limited effect on the extensional viscosity in an extensional flow. Hence, we expect that a silica suspension is expected to show only a slight extensional thickening behavior. Two different kinds of silica, Aerosil 200 and COK 84 (Evonik Degussa), have been used. Aerosil 200 is more suitable to form flocculated suspensions in nonpolar fluids. It was used to form the test fluid PG1: paraffin $+4 \%$ Aerosil 200. The other kind of Aerosol is COK 84 that is suitable also for polar fluids. It was used to form the test fluid EG2: ethanol $+10 \%$ COK 84. 
From a rheological point of view, the fluids LP5, EG1, CP1, PG1, and EG2 could be classified as gels having a distinct yield stress and shear thinning behavior.

\section{RESULTS}

\subsection{Boger Fluids}

The rheological characterization of the three boger fluids tested (BF1-BF3) has been conducted by Mun et al. [6]. The values of shear viscosity and surface

Table 2 Shadowgraph images of the spray test conducted with three different Boger fluids

\begin{tabular}{|c|c|c|c|c|c|}
\hline Boger fluid & $\begin{array}{l}\text { Low } \\
\text { velocity } \\
\text { range } \\
\end{array}$ & $\begin{array}{l}\text { Medium } \\
\text { velocity } \\
\text { range } \\
\end{array}$ & $\begin{array}{c}\text { High } \\
\text { velocity } \\
\text { range } \\
\end{array}$ & $\begin{array}{c}\text { Shear } \\
\text { viscosity, } \\
\mathrm{mPa} \cdot \mathrm{s} \\
\end{array}$ & $\begin{array}{c}\text { Surface } \\
\text { tension, } \\
\mathrm{mN} / \mathrm{m} \\
\end{array}$ \\
\hline $\begin{array}{c}\text { BF1 } \\
\text { Low } \\
\text { molecular } \\
\text { weight PEO } \\
\text { solution } \\
M_{w}=1 \cdot 10^{5} \mathrm{~g} / \mathrm{mol}\end{array}$ & $\begin{array}{c}u_{j}=5.8 \mathrm{~m} / \mathrm{s} \\
\operatorname{Re}=817\end{array}$ & $\begin{array}{c}u_{j}=19.5 \mathrm{~m} / \mathrm{s} \\
\operatorname{Re}=2750\end{array}$ & $\begin{array}{c}u_{j}=70.2 \mathrm{~m} / \mathrm{s} \\
\operatorname{Re}=9895\end{array}$ & 5.25 & 71.9 \\
\hline $\begin{array}{c}\text { BF2 } \\
\text { Medium } \\
\text { molecular } \\
\text { weight PEO } \\
\text { solution } \\
M_{w}=3 \cdot 10^{5} \mathrm{~g} / \mathrm{mol}\end{array}$ & $\begin{array}{c}u_{j}=6.5 \mathrm{~m} / \mathrm{s} \\
\operatorname{Re}=944\end{array}$ & $\begin{array}{c}u_{j}=20.8 \mathrm{~m} / \mathrm{s} \\
\operatorname{Re}=3020\end{array}$ & $\begin{array}{c}u_{j}=70.2 \mathrm{~m} / \mathrm{s} \\
\operatorname{Re}=10,200\end{array}$ & 5.20 & 71.2 \\
\hline $\begin{array}{c}\text { BF3 } \\
\text { High } \\
\text { molecular } \\
\text { weight PEO } \\
\text { solution } \\
M_{w}=1 \cdot 10^{6} \mathrm{~g} / \mathrm{mol}\end{array}$ & $\begin{array}{c}u_{j}=5.2 \mathrm{~m} / \mathrm{s} \\
\operatorname{Re}=825\end{array}$ & $\begin{array}{c}u_{j}=19.5 \mathrm{~m} / \mathrm{s} \\
\operatorname{Re}=3090\end{array}$ & $\begin{array}{c}u_{j}=69.8 \mathrm{~m} / \mathrm{s} \\
\operatorname{Re}=1170\end{array}$ & 4.75 & 71.0 \\
\hline
\end{tabular}


tension are shown in Table 2. The values of shear viscosity for the three fluids are comparable and around $5 \mathrm{mPa} \cdot \mathrm{s}$. Also, the values of surface tension are very similar and around $71 \mathrm{mN} / \mathrm{m}$. The extensional viscosity of these fluids has been measured by Mun et al. [6] with a Rheometrics RFX opposed jet rheometer, and the results are shown in Fig. 4. The fluid with the lower molecular weight (BF1) shows only a very slight increase in the Trouton ratio for increasing shear rate. On the other hand, the increase in Trouton ratio for the fluid with the higher molecular weight (BF3) is much more significant.

The shadowgraph images of the spray tests conducted with the Boger fluids are shown in Table 2. The test fluid BF1 at low velocity forms a liquid sheet flapping in the lower part. The upper part of the sheet is surrounded by a rim. The rim is unsta-

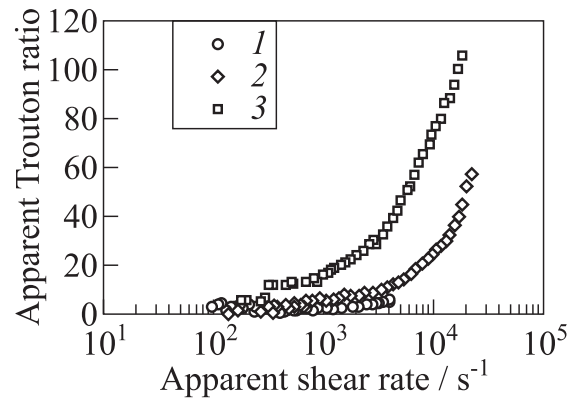

Figure 4 Apparent Trouton ratio values for three different Boger fluids (data from [6]): $1-\mathrm{BF} 1 ; 2-\mathrm{BF} 2$; and $3-$ BF3

ble with shedding of droplets. At medium injection velocity, the waves can be observed in the liquid sheet. In the high velocity range, a liquid sheet cannot be observed: the fluid breaks up into droplet very close to the impingement point.

The behavior of BF2 at low velocity is similar to BF1. The disturbances of the rim lead to the formation of long filaments detaching from the rim. A rim can also be observed in the medium velocity range. The sheet shows strong flapping. At high injection velocity, the rim disappears. The liquid sheet is still present and it breaks up into transversal ligaments. These ligaments are fragmented into smaller threads further downstream.

At low injection velocity, the fluid BF3 forms a sheet completely surrounded by a rim. The rim shows some disturbances but does not break up. At increasing injection velocity, the rim becomes more unstable and the waves are clearly visible in the sheet. In the high velocity range, the rim disappears. The liquid sheet breaks up into threads.

\subsection{Linear Polymer Solutions}

The results of the spray tests conducted with solutions of water and Methocel J75MS at increasing concentrations are shown in Table 3. The solution with the lower concentration (LP1) at low velocity shows a liquid sheet completely surrounded by a rim. The rim shows some disturbances but do not break up into droplets. In the medium velocity range, the rim disappears and the sheet breaks 
Table 3 Shadowgraph images of the spray tests conducted with solutions of water and Methocel J75MS $\left(M_{n}=220,000\right)$ with increasing concentrations

\begin{tabular}{cccc}
\hline Solution & Low velocity range & Medium velocity range & High velocity range \\
\hline LP1 & & & \\
Water \\
$+0.5 \%$ \\
Methocel \\
J75MS
\end{tabular}

up into ligaments. At a high injection velocity, only a small disturbed sheet can be observed. The sheet ruptures into ligaments that break up into smaller parts further downstream.

The solution LP2 shows at the low injection velocity a very small sheet surrounded by a stable rim. The rim disappears at higher injection velocity. In the middle velocity range, the sheet is quite wide. The rupture of the sheet is caused by a flapping that leads to the formation of holes and ligaments. At high injection velocity, the behavior is similar with the formation of thinner threads.

The behavior of the fluid LP3 at low velocity is similar to that of LP2. In the medium velocity range, the liquid sheet produced by LP3 is very broad. A rim can still be observed in the upper part. The sheet shows very strong flapping in the lower part, but the disturbances lead only to a folding of the sheet without 
Table 4 Shadowgraph images of the spray tests conducted with solutions of water and Methocel with increasing molecular weights

\begin{tabular}{|c|c|c|c|}
\hline Solution & Low velocity range & Medium velocity range & High velocity range \\
\hline \multirow{4}{*}{$\begin{array}{c}\text { LP4 } \\
\\
\text { Water } \\
+2 \% \\
\text { Methocel } \\
\text { F50 } \\
M_{n}=20,000\end{array}$} & \multirow[b]{3}{*}{$20 \mathrm{~mm}$} & & \\
\hline & & & \\
\hline & & & \\
\hline & $u_{j}=5.2 \mathrm{~m} / \mathrm{s}$ & $u_{j}=28.6 \mathrm{~m} / \mathrm{s}$ & /s \\
\hline$\overline{L P 5}$ & 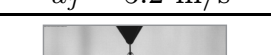 & $\bar{Y}$ & \multirow{6}{*}{ 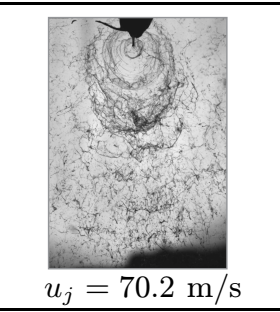 } \\
\hline \multirow{5}{*}{$\begin{array}{c}\text { Water } \\
+2 \% \\
\text { Methocel } \\
\text { F4M } \\
M_{n}=86,000\end{array}$} & \multirow{5}{*}{$u_{j}=6.5 \mathrm{~m} / \mathrm{s}$} & & \\
\hline & & & \\
\hline & & & \\
\hline & & & \\
\hline & & $u_{j}=27.3 \mathrm{~m} / \mathrm{s}$ & \\
\hline LP3 & $Y$ & \multirow[t]{5}{*}{$\gamma$} & \multirow{6}{*}{$\begin{array}{l}\left(\begin{array}{l}1 \\
3 \\
5\end{array}\right) \\
u_{j}=70.2 \mathrm{~m} / \mathrm{s}\end{array}$} \\
\hline \multirow{5}{*}{$\begin{array}{c}\text { Water } \\
+2 \% \\
\text { Methocel } \\
\text { J75MS } \\
M_{n}=220,000\end{array}$} & \multirow{5}{*}{$u_{j}=6.5 \mathrm{~m} / \mathrm{s}$} & & \\
\hline & & & \\
\hline & & & \\
\hline & & & \\
\hline & & $u_{j}=29.9 \mathrm{~m} / \mathrm{s}$ & \\
\hline
\end{tabular}

ruptures in the central part. The formation of holes can be observed in the lateral portions. At high injection velocity, the sheet ruptures into thick threads. These threads do not break up downstream.

The results of the spray tests conducted with solutions of water and different kinds of Methocel with increasing molecular weight are shown in Table 4.

The solution LP4 at low velocity produces a sheet surrounded by a rim. The rim shows strong instabilities. The waves are also clearly visible in the liquid sheet. At higher injection velocities, the rim disappears. The liquid sheet breaks up into transversal ligaments that rupture into droplets downstream. At high injection velocity, the liquid sheet is small and ruffled. Here, the breakup into droplets is close to the impingement point.

The behavior of LP5 at low injection velocity is similar to LP2 and LP3. A small sheet surrounded by a stable rim can be observed. Increasing the injection velocity, it can be seen that a large sheet is formed. No rim can be observed 
in the medium velocity range. The sheet is smooth near the impingement point but near the edge, a flapping of the sheet can be observed. This instability leads to a folding of the sheet on itself and, eventually, to a rupture. In the high velocity range, the sheet breaks up into smaller threads.

\subsection{Crosslinked Polymer Solution}

The shadowgraph images obtained with the crosslinked polymer solution CP1 are shown in Table 5. At low injection velocity, the formed liquid sheet is very small. Increasing the injection velocity, a small liquid sheet can be observed that breaks up into ligaments. These ligaments disrupt into large pieces in the central part of the spray, while at the side of the spray, smaller pieces are produced. At high injection velocity, the breakup of the sheet leads to formation of drops.

Table 5 Shadowgraph images of the spray tests with a crosslinked polymer solution

\begin{tabular}{|c|c|c|c|c|}
\hline Solution & $\begin{array}{c}\text { Low velocity } \\
\text { range }\end{array}$ & $\begin{array}{l}\text { Medium velocity } \\
\text { range }\end{array}$ & $\begin{array}{c}\text { High velocity } \\
\text { range }\end{array}$ & $\begin{array}{c}\text { Yield } \\
\text { stress, Pa }\end{array}$ \\
\hline $\mathrm{CP} 1$ & & & & \\
\hline $\begin{array}{c}\text { Water } \\
+1 \% \\
\text { Carbopol } \\
980\end{array}$ & & $\frac{7}{3}$ & & 150 \\
\hline & $u_{j}=6.5 \mathrm{~m} / \mathrm{s}$ & $u_{j}=27.3 \mathrm{~m} / \mathrm{s}$ & $u_{j}=70.2 \mathrm{~m} / \mathrm{s}$ & \\
\hline
\end{tabular}

\subsection{Silica Suspensions}

The shadowgraph images of the spray tests conducted with the silica suspensions are shown in Table 6. The generalized Reynolds numbers in this table are based on the Herschel Bulkley Extended (HBE) law. More details on this constitutive law can be found in [23].

The suspension PG1 at low injection velocity forms a very elongated flat sheet surrounded by a thick rim. At higher injection velocities, the rim disappears. The liquid sheet is relatively small in the medium velocity range and ruffled. The sheet breaks up through transversal ligaments that disintegrate into droplets. At high velocity, the sheet is very small and the ligaments appear close to the impingement point. The ligaments break up into drops close to the impingement point.

The suspension EG2 shows only a small unstable sheet at low injection velocity. The small liquid sheet breaks up into transversal ligaments that disintegrate 
Table 6 Shadowgraph images of the spray tests conducted with silica suspensions (images from [2])

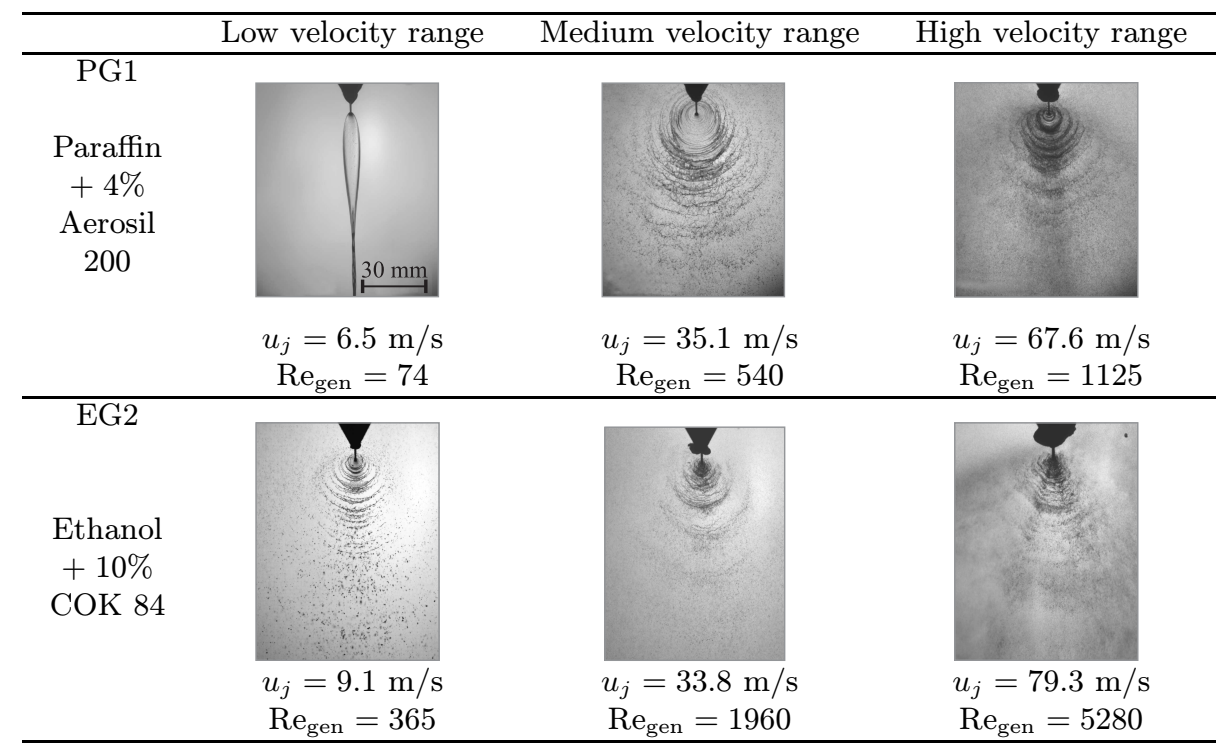

into droplets downstream. At high injection velocity, no liquid sheet can be observed and the rupture of ligaments into droplets takes place close to the impingement point.

\subsection{Ethanol Gels}

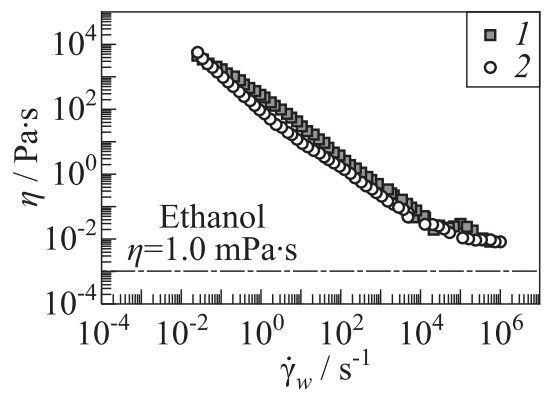

Figure 5 Comparison of the shear viscosity of the ethanol gels (data from [2]): 1 - EG1 (Methocel) and $2-\mathrm{EG} 2(\mathrm{COK})$
As an example for gels with similar viscosity curves, two different ethanol gels (EG1 and EG2) have been studied. The flow curves for these fluids are shown in Fig. 5. The measurements have been conducted with a rotational rheometer with a cone-plate sensor in the low shear rate range (up to $10^{4} \mathrm{~s}^{-1}$ ) and with a capillary rheometer in the upper range. The results show that the two tested ethanol-based gels have 
Table 7 Shadowgraph images of the spray tests and comparison of the shear viscosity of the ethanol gels (images from [2])

\begin{tabular}{|c|c|c|c|c|}
\hline Fluid & $\begin{array}{l}\text { Low velocity } \\
\text { range }\end{array}$ & $\begin{array}{l}\text { Medium velocity } \\
\text { range }\end{array}$ & $\begin{array}{l}\text { High velocity } \\
\text { range }\end{array}$ & $\begin{array}{c}\text { Yield } \\
\text { stress, Pa }\end{array}$ \\
\hline \multirow[t]{2}{*}{$\begin{array}{c}\text { Ethanol } \\
+3.5 \% \\
\text { Methocel } 311\end{array}$} & \multirow[t]{2}{*}{ No impingement } & 5 & 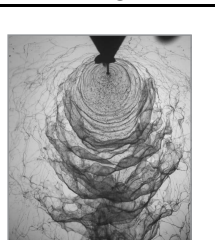 & \multirow[t]{2}{*}{360} \\
\hline & & $\begin{array}{c}u_{j}=31.2 \mathrm{~m} / \mathrm{s} \\
\mathrm{Re}_{\mathrm{gen}}=894\end{array}$ & $\begin{array}{c}u_{j}=76.7 \mathrm{~m} / \mathrm{s} \\
\operatorname{Re}_{\text {gen }}=2290\end{array}$ & \\
\hline \multirow[t]{2}{*}{$\begin{array}{c}\text { Ethanol } \\
+10 \% \\
\text { COK } 84\end{array}$} & 8 & g & & \multirow[t]{2}{*}{120} \\
\hline & $\begin{array}{c}u_{j}=9.1 \mathrm{~m} / \mathrm{s} \\
\text { Re }_{\text {gen }}=365\end{array}$ & $\begin{array}{c}u_{j}=33.8 \mathrm{~m} / \mathrm{s} \\
\operatorname{Re}_{\text {gen }}=1960\end{array}$ & $\begin{array}{c}u_{j}=79.3 \mathrm{~m} / \mathrm{s} \\
\operatorname{Re}_{\text {gen }}=5280\end{array}$ & \\
\hline
\end{tabular}

very similar shear viscosities for a wide range of shear rates $\left(10^{-1}-10^{6} \mathrm{~s}^{-1}\right)$. The yield stresses have also been determined, and the results are given in Table 7 .

The results of the spray tests conducted with EG1 and EG2 are shown in Table 7. When conducting tests with EG1 at low injection velocity, the two jets do not impinge. In the medium velocity range, the gel EG1 produces a liquid sheet surrounded by a rim. The sheet shows strong flapping in the lower part. At high injection velocity, a broad sheet can be observed. The flapping of the sheet leads to the formation of thread structures.

The spray behavior of EG2 has been already described in subsection 4.4.

\subsection{Capillary Rheometer Measurements}

Four of the fluids used in the spray tests have been characterized with a capillary rheometer. The fluids used are: EG1, LP3, CP1, and EG2. The measurements obtained with the capillary rheometer are shown in Fig. 6. The measurements with the capillary rheometer have been conducted to cover an apparent shear rate range from $1 \cdot 10^{5}$ to $2 \cdot 10^{6} \mathrm{~s}^{-1}$. For each apparent shear rate, the measurements with both an orifice (0.3-millimeter diameter) and a capillary (0.3-millimeter 


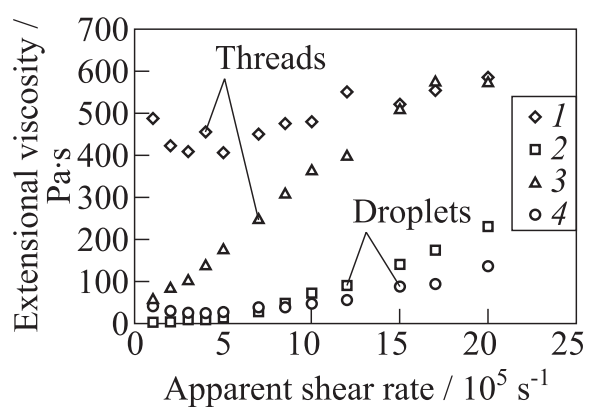

(a)

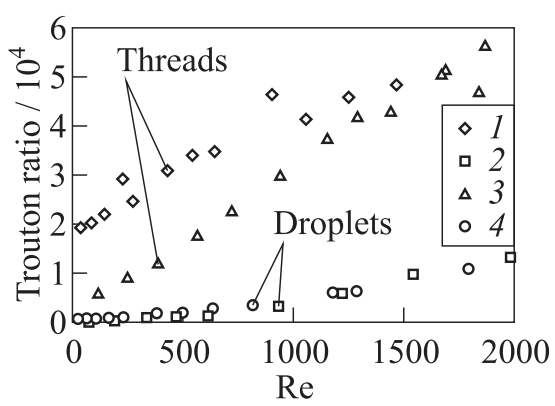

(b)

Figure 6 Extensional viscosity $(a)$ and Trouton ratio $(b)$ measured with the capillary rheometer. In the graphs, it is also noted which fluids formed thread and which formed droplets during the spray tests: 1 - EG1 (ethanol + 3.5\% Methocel 311); 2 - EG2 (ethanol $+10 \%$ COK 84$) ; 3-$ LP3 (water $+2 \%$ Methocel J75MS); and $4-$ CP1 (water $+1 \%$ Carbopol 980)

diameter and 16-millimeter length) have been conducted. With these results, it is possible to apply Bagley and Rabinowitsch correction to obtain a more accurate value of the shear viscosity. Applying Cogswell theory, it is also possible to determine the elongational viscosity. Figure $6 a$ shows the results in terms of extensional viscosity vs. apparent shear rate. The results can also be presented as nondimensional numbers as shown in Fig. $6 b$. This can be done because the capillary rheometer gives for each measurement the corrected shear viscosity, the elongational viscosity, and the fluid velocity in the capillary. It is, therefore, possible to determine for each measurement the Trouton ratio and the Reynold number. The extensional viscosities of both EG1 and LP3 are much larger than those for the other two fluids CP1 and EG2 in the high apparent shear rate range. When the results are expressed in terms of nondimensional numbers (see Fig. $6 b$ ), the difference between the Trouton ratio of the fluids EG1 and LP3 and the Trouton ration of fluids CP1 and EG2s is marked on the entire range of Reynolds numbers investigated.

\section{DISCUSSION}

\subsection{Spray Tests}

The tests with Boger fluids show that the fluid with the higher molecular weight (BF3) leads to the formation of very pronounced threads. These threads do not break up into droplets even at the higher injection velocities. On the other hand, 
the behavior of the fluid with the lower molecular weight (BF3) resembles the behavior of a Newtonian fluid, with breakup into droplets already occurring at low speeds. In the tests with linear polymer solutions, it can be observed that the most concentrated solution of the polymer with the higher molecular weight (LP3) is the one that produces the most marked threads. These threads do not break up even at high injection velocities. More dilute solutions of the same polymer (LP1 and LP2) still show the formation of threads at lower velocities; however, with increasing injection velocity, these threads have a tendency to break up.

Thread formation is not observed for the polymer solution with the lower molecular weight (LP4). The behavior of this solution at high injection velocity resembles that of a Newtonian fluid. The crosslinked polymer solution (CP1) tested leads to the formation of relatively large structures at low and intermediate injection velocities. At higher velocities, the fluid breaks up into droplets. The formation of marked threads is not observed. Both silica suspensions tested lead to the formation of droplets when sprayed with an impinging jet injector, while thread formation is not observed.

The results from all the tested fluids confirm that a correlation seems to exist between thread formation and the extensional behavior of the fluids. In particular, fluids with high values of the Trouton ratio (in the high shear rate range) formed marked threads. In the case of the tested Boger fluids, direct measures of the Trouton ratio are available from [6]. These measures show that increasing the molecular weight increases the Trouton ratio. The fluid with the higher Trouton ratio is the one producing the most marked threads which break up less also at high injection velocities. As previously explained for linear polymer solutions, measurements of elongational behavior are not available in literature. From the considerations done in section 2, an increase in molecular weight is expected to lead to an increase in the Trouton ratio. The spray experiments in Table 4 show how fluids with higher molecular weight produce more marked threads. The considerations of section 2 can be applied also to silica suspensions. A silica suspension is expected to show only a slight extensional thickening behavior. This agrees with the results from the spray tests: the tested colloidal suspensions break up into droplets while thread formation is not observed.

The influence of other fluid properties on thread formation could also be determined from the tests conducted. To determine if a correlation between shear viscosities and thread formation exists, the results of the tests with the two ethanol gels (see subsection 4.5), are particularly significant. The shear viscosities of the two gels are very similar in the range of shear rates $10^{-1}$ to $10^{6} \mathrm{~s}^{-1}$, but the spray behavior is completely different: the gel with Methocel (a linear polymer) produces very marked threads, while the gel using COK 84 (fumed silica) produces droplets. A similar observation can be made considering the tests conducted with the Boger fluids (see subsection 4.1): the three fluids have very similar shear viscosities, but behave differently concerning thread forma- 
tion. From these results, it seems that shear viscosity does not play a significant role in thread formation.

The possible influence of surface tension on thread formation has been studied for the tests with Boger fluids. The surface tensions of Boger fluids have been measured and it was found that they are very similar. However, BF1 leads to formation of droplets, BF2 to the formation of weak threads, and BF3 to the formation of marked threads. Therefore, for the tests with these kinds of Boger fluids, thread formation is not influenced by surface tension. However, the influence of surface tension should be studied in future works considering a wider range of fluids.

\subsection{Capillary Rheometer Measurements}

The goal of the tests is to determine if a correspondence between the values of elongational viscosity obtained with the capillary rheometer and the formation of threads exists. The spray behavior of the fluids tested can be summarized as follows: EG1 leads to very marked thread formation and also with LP3 thread formation can be observed. On the other hand, the drops were formed in the spray tests conducted with CP1 and EG2. The results of the measurements conducted with the capillary rheometer show that the extensional viscosities of both EG1 and LP3 are much larger than those for the other two fluids CP1 and EG2 in the high shear rate range. This relates well with the fact that marked thread formation at high injection velocities is observed only with EG1 and LP3. When the results are expressed in terms of nondimensional numbers (see Fig. $6 b$ ), the difference of Trouton ratio between fluids producing threads (EG1 and LP3) and fluids producing droplets is marked on the entire range of Reynolds numbers investigated. One can conclude that a distinct correlation seems to exist between thread formation and the value of the extensional viscosity at high apparent shear rates. Finally, a consideration can be made on the possibility of obtaining quantitative values of elongational viscosity using the Cogswell theory. The numerical values of the Trouton ratio at high shear rates are very high (above 10000 ) also for CP1 (water $+1 \%$ Carbopol). This does not agree with the results from Yarin et al. [24]. They tested an aqueous solution of Carbopol with a capillary thinning rheometer and measured values of the Trouton ratio close to 3 , which means that such a solution is not elastic.

\section{SUMMARY AND CONCLUDING REMARKS}

The formation of threads-like structures instead of droplets was observed in recent studies on the injection of gels and other non-Newtonian fluids through impinging jet injectors. To obtain a better understanding of the phenomenon of 
thread formation, a research campaign is currently ongoing at DLR. Tests with different classes of non-Newtonian fluids have been conducted. The results from all the tested fluids confirm that a correlation seems to exist between thread formation and the extensional behavior of the fluids. In particular, fluids with high values of the Trouton ratio (in the high shear rate range) formed marked threads. The measurement of the extensional viscosity for fluids with a distinct yield stress is problematic. In the present work, the extensional behavior has been determined by applying Cogswell theory to the measurements obtained with a capillary rheometer. The results obtained with this technique show good agreement with the spray tests conducted: a higher Trouton ratio corresponds to a more marked thread formation.

\section{REFERENCES}

1. Von Kampen, J., H. K. Ciezki, T. Tiedt, and K. Madlener. 2006. Some aspects of the atomization behavior of Newtonian and of shear-thinning gelled non-Newtonian fluids with an impinging jet injector. 42nd AIAA Joint Propulsion Conference. Sacramento, CA, USA.

2. Madlener, K. 2008. Die Strömungs- und Versprühungseigenschaften gelförmiger Fluide. Ph.D. Thesis.

3. Miller, E., B. Gibson, E. McWilliams, and J. P. Rothstein. 2005. Collision of viscoelastic jets and the formation of fluid webs. Appl. Phys. Lett. 87.

4. Coil, M. A. 2009. Effect of viscoelastic properties in impinging jet sprays. ICLASS 2009, 11th Triennial Annual Conference (International) on Liquid Atomization and Spray Systems. Vail, CO, USA.

5. McCabe, J.D., and M. A. Coil. 2010. A graphical spray analysis method for gel spray characterization. 46th AIAA/ASME/SAE/ASEE Joint Propulsion Conference \& Exhibit. Nashville, TN, USA.

6. Mun, R. P., J. A. Byars, and D. V. Boger. 1998. The effects of polymer concentration and molecular weight on the breakup of laminar capillary jets. J. Non-Newtonian Fluid Mech. 74:285-97.

7. Christanti, Y., and L.M. Walker. 2001. Surface tension driven jet break up of strain-hardening polymer solutions. J. Non-Newtonian Fluid Mech. 100:9-26.

8. Christanti, Y., and L. M. Walker. 2002. Effect of fluid relaxation time of dilute polymer solutions on jet breakup due to a forced disturbance. J. Rheology 46:73348.

9. Chao, K. K., C. A. Child, E. A. Grens II, and M. C. Williams. 1984. Antimisting action of polymeric additives in jet fuels. AlChE J. 30:112-20.

10. Mansour, A., and N. Chigier. 1995. Air-blast atomization of non-Newtonian liquids. J. Non-Newtonian Fluid Mech. 58:161-94.

11. Mun, R.P., B.W. Young, and D. V. Boger. 1999. Atomisation of dilute polymer solutions in agricultural spray nozzles. J. Non-Newtonian Fluid Mech. 83:163-78. 
12. Thompson, J. C., and J. P. Rothstein. 2007. The atomization of viscoelastic fluids in flat-fan and hollow-cone spray nozzles. J. Non-Newtonian Fluid Mech. 147:11-22.

13. Harrison, G. M., R. P. Mun, G. Cooper, and D. V. Boger. 1999. A note on the effect of polymer rigidity and concentration on spray atomisation. J. Non-Newtonian Fluid Mech. 85:93-104.

14. Stelter, M., G. Brenn, and F. Durst. 2002. The influence of viscoelastic fluid properties on spray formation from flat-fan and pressure-swirl atomizers. Atomization Sprays 12:299-327.

15. Negri, M., and H.K. Ciezki. 2010. Atomization of non-Newtonian fluids with an impinging jet injector: Influence of viscoelasticity on hindering droplets formation. 46th AIAA/ASME/SAE/ASEE Joint Propulsion Conference \& Exhibit. Nashville, TN, USA.

16. Niedzwiedz, K., O. Arnolds, N. Willenbacher, and R. Brummer. 2009. How to characterize yield stress fluids with capillary breakup extensional rheometry (CaBER). Appl. Rheol. 19.

17. Rajagopalan, D. 2000. Computational analysis of techniques to determine extensional viscosity from entrance flows. Rheologica Acta 39:138-51.

18. Macosko, C.W. 1993. Rheology — principles, measurements and applications. Wiley-VCH.

19. Barnes, H.A. 2000. A handbook of elementary rheology. Institute of NonNewtonian Fluid Mechanics University of Wales.

20. Perkins, T. T., D.E. Smith,, and S. Chu. 1997. Single polymer dynamics in an elongational flow. Science 276:2016-21.

21. Raghavan, S.R., and S.A. Khan. 1997. Shear-thickening response of fumed silica suspensions under steady and oscillatory shear. J. Colloid Interface Sci. 185:57-67.

22. Chellamuthu, M., E. M. Arndt, and J. P. Rothstein. 2009. Extensional rheology of shear-thickening nanoparticle suspensions. Soft Matter. 5:2117-24.

23. Madlener, K., and H.K. Ciezki. 2009. Some aspects of rheological and flow characteristics of gel fuels with regard to propulsion application. 45th AIAA/ASME/SAE/ASEE Joint Propulsion Conference \& Exhibit. Denver, CO, USA.

24. Yarin, A. L., E. Zussman, and A. Theron. 2004. Elongational behavior of gelled propellant simulants. J. Rheology 48:101-16. 